\title{
An adult model of preschool children's speech memory
}

\author{
NELSON COWAN, CRISTY CARTWRIGHT, CARRIE WINTEROWD, and MOLLY SHERK \\ University of Missouri, Columbia, Missouri
}

\begin{abstract}
In previous work, it has been demonstrated that phonetic similarity among the items in a spoken list interferes with recall much more in school-aged children than in preschool children. The basis of this developmental change, however, is unclear. In the present study we examined the possibility that a developmental increase in the use of covert verbal rehearsal accounts for the change in the effects of phonetic similarity. Adults who recalled lists of spoken words during articulatory suppression tasks that blocked covert rehearsal were found to display patterns of recall that resembled those ordinarily found in 5-year-old children. The specific aspects of rehearsal responsible for these effects also were investigated.
\end{abstract}

There are developmental changes during childhood in various processing mechanisms that assist in memory for speech. Our knowledge of these processes often is not extensive enough to determine which mechanisms account for the developmental changes observed in a particular memory task. An important example is the phonetic similarity effect first observed by Conrad (1971), who presented children with series of pictures with names that sounded similar (e.g., hat, bat, cat, etc.) or dissimilar (e.g., fish, clock, spoon, etc.). School-aged children remembered series of pictures with dissimilar names much better than series with similar names, whereas for preschool children the similarity of the names made much less difference. (Younger children's memory performance also was poorer overall, so the developmental change was primarily an increase in memory for dissimilar lists.) Conrad suggested that the observed age differences resulted from the development of some form of covert verbal labeling of the pictures, which presumably is of more mnemonic value when the items' names are dissimilar. Conrad's results were replicated by Hulme (1984) in an experiment in which the stimuli were spoken without accompanying pictures. These experiments, however, provide no direct support for the suspected role of covert speech.

A study conducted by Hayes and Rosner (1975) with 4- and 5-year-old children suggested that the developmental change in the effects of phonetic similarity might result from an increase with age in the amount of cumulative rehearsal of list items (i.e., a particular type of covert speech). Hayes and Rosner used a procedure similar to that used by Conrad (1971), except that two verbal training conditions were included. In one of these, the child

This research was supported by NIH Grant 1-R23-HD21338-02 awarded to the senior author. We thank Scott Saults and Jean Ispa for comments on an earlier draft. Address reprint requests to Nelson Cowan, Department of Psychology, University of Missouri, 210 McAlester Hall, Columbia, MO 65211 . learned to name each picture only once as it was presented. In the other, the child learned to rehearse the items cumulatively; all of the items already presented were named whenever a new item was presented. A phonetic similarity effect was observed in the rehearsal condition, but not in the label-once or control conditions.

Although these results suggest that the development of rehearsal may underlie increases with age in the phonetic similarity effect, the observed effect of rehearsal training conceivably could be misleading. It is possible that young children are deficient in some other memory mechanism but that trained rehearsal somehow compensates for the true deficiency. Alternative memory mechanisms are not entirely hypothetical: developmental changes have been observed in studies of auditory sensory memory (Cowan, Suomi, \& Morse, 1982; Irwin, Ball, Kay, \& Stillman, 1985; Sipe \& Engle, 1986) and speech representation (Cowan, Braine, \& Leavitt, 1985; Cowan \& Kielbasa, 1986; Morais, Carey, Alegria, \& Bertelson, 1979; Read, 1978; Treiman, 1985). The trained rehearsal may act to strengthen the representation in one of these passive types of memory, even if older subjects would not need to rehearse in order for a stronger speech representation to be formed.

Convergent evidence on the role of covert speech in memory within older subjects would help to resolve this question, and in the present study we examined one such source of evidence. Covert speech processes in adults can be blocked with a technique termed "articulatory suppression" (Murray, 1967). In this technique, the subject repeats a single word or phrase while trying to learn a list of words. In the most relevant of these studies, Murray (1968) and Peterson and Johnson (1971) presented visual and auditory series of rhyming or nonrhyming letters for serial recall, with or without articulatory suppression concurrent to the presentation. They found that there was a detrimental effect of phonetic similarity with either visual or auditory presentation of the letters. This effect was eliminated by articulatory suppression when the lists were 
presented visually, but not when they were presented acoustically.

The presence of an effect of phonetic similarity for spoken lists even during articulatory suppression may appear to contradict the hypothesis that the similarity effect depends upon rehearsal. Actually, though, the situation may be complex: there may be two separate components of the similarity effect, only one of which depends upon rehearsal. The other component would be acoustic or phonetic confusion within a passive store that persists for a time even without rehearsal. Perhaps because a nonrehearsal component of the similarity effect exists, a small effect of phonetic similarity is sometimes obtained even in 4- and 5-year-old subjects (see Alegria \& Pignot, 1979; Hulme, 1984).

If there are two bases of the phonetic similarity effect, we would expect articulatory suppression to result in a reduction, but not necessarily an elimination, of the effect. However, the methods of scoring and data analysis in the adult studies of Murray (1968) and Peterson and Johnson (1971) were not sufficient to determine whether articulatory suppression caused a reduction in the phonetic similarity effect for auditory stimuli. Furthermore, the articulatory suppression task in both studies involved speaking aloud, and the resulting auditory interference conceivably could have altered the magnitude of the phonetic similarity effect. In another adult study, Levy (1971) used a silent articulatory suppression task, but she obtained no significant reduction of the phonetic similarity effect for stimulus lists in either modality.

In order to examine the possible contribution of rehearsal to the developmental differences that have been observed, in the present adult study we combined a methodology comparable to that of Hulme (1984) with an articulatory suppression task that was whispered rather than spoken aloud. A whispered task should allow the experimenter to check the subject's articulations, while greatly reducing any auditory interference. To the extent that developmental differences in the phonetic similarity effect and the level of recall are based on rehearsal, adults carrying out whispered articulatory suppression tasks should recall spoken lists in a manner resembling that of young children.

\section{EXPERIMENT 1}

\section{Method}

Subjects. The subjects were 32 college students ( 10 men and 22 women) who received credit in introductory psychology courses. They were native English speakers with no known speech or hearing defects.

Stimuli. The stimulus words were the same as those used by Hulme (1984), which were also the labels to the pictures used by Conrad (1971). The phonetically similar word lists were composed of subsets of the words rat, cat, mat, hat, bat, man, bag, tap, and the dissimilar lists were composed of subsets of the words girl, bus, train, spoon, fish, horse, clock, hand. The presentation rate was one item per second. The stimuli were presented orally rather than taped in this experiment, in order to replicate the procedure used in the developmental studies (e.g., Hulme, 1984). An oral presentation typically is used in developmental studies because young children sometimes do not attend well to taped presentations.

Procedure. Experimentation was conducted with each subject individually, in a quiet room. First, the subject heard and repeated all 16 of the stimulus words to ensure that the words were correctly perceived. This was followed by two experimental conditions, presented in either order: an articulatory suppression condition, in which the subject whispered the alphabet (as quickly as possible without making mistakes) while listening to each list of words, and a control condition, in which the subject listened quietly to each list. Before each of these conditions there was a practice trial with three items that were not from the test lists ( foot, knife, and cow for the first condition, and bear, sun and glass for the second condition). A phonetically similar practice set was not included, because the developmental studies did not use one. Because it seems unlikely that a phonetically similar practice set could somehow enhance the effects of articulatory suppression, its omission should not work in favor of the main hypothesis that the similarity effect can be reduced using articulatory suppression.

The procedure for the control condition was similar to that used by Hulme (1984). The subject listened silently while a list was read and then immediately attempted to repeat the items on the list in order. The procedure for the articulatory suppression condition was similar, except that the subject whispered the alphabet as rapidly as possible starting about $2 \mathrm{sec}$ before each list began and continuing until the recall cue. In both conditions, the experimenter always made a hand motion as a cue to recall, $1 \mathrm{sec}$ following the last list item. Phonetically similar and dissimilar word lists were presented in alternating order, with half of the subjects receiving a similar-word list first and half receiving a dissimilar-word list first. Memory span for each type of list was determined by presenting lists of increasing length. The first two lists of each type contained three words, drawn randomly from the eight possibilities. If the subject correctly repeated at least one of the two lists of a particular type, progressively longer lists of that type would be presented until the subject erred on both trials of the same list length. Similarly, testing on the other type of list continued until the subject erred on both trials of one length. The subject's word span for each list type (phonetically similar or dissimilar) was the longest list-length of that type repeated successfully. As in the studies of Conrad (1971) and Hulme (1984), subjects received credit only if they repeated the words in the correct serial order.

For every subject, the alternating order of phonetically similar and dissimilar trials was the same within the two articulation conditions. However, different word lists were used for a subject's first and second conditions, and no particular word list was presented more than once to a subject.

\section{Results and Discussion}

The mean word spans in all conditions of this experiment are shown in the second panel of Figure 1; the results of Hulme (1984), drawn from his tabulated data, are depicted for comparison in the first panel of the figure. It is apparent that both the level of performance and the magnitude of the phonetic similarity effect were reduced by the articulatory suppression task. Whereas the adults' pattern of performance in the control condition was quite similar to that of Hulme's 10-year-old subjects, their performance in the articulatory suppression condition was most similar to that of the 5-year-old children.

The effects of articulatory suppression were assessed by an ANOVA with the articulation condition (control vs. 


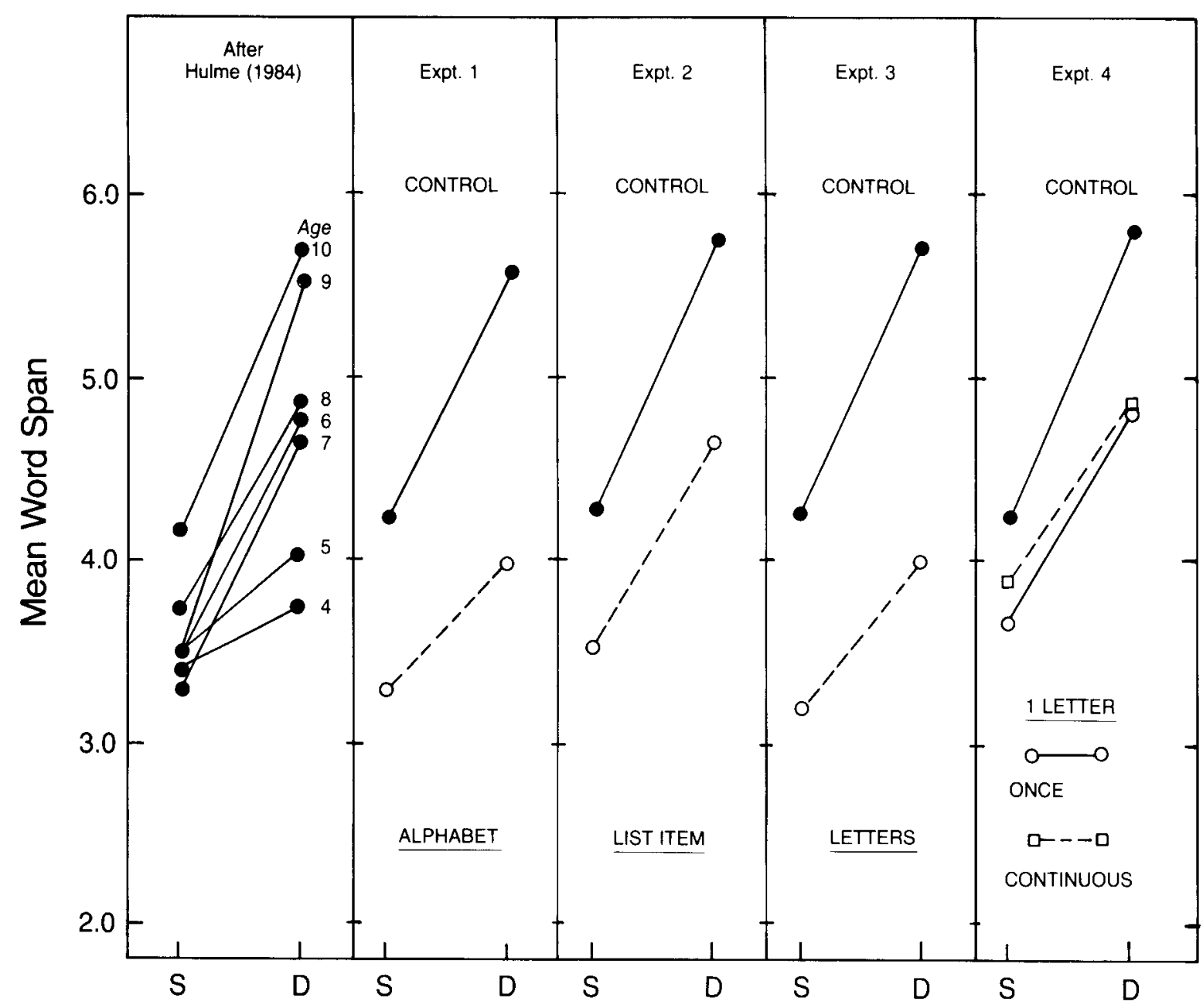

List Type: Phonologically Similar (S) or Dissimilar (D)

Figure 1. Mean word span for children of each age in Hulme's (1984) study (leftmost panel) and for adults in each of the four experiments within the present study, for phonetically similar (S) and dissimilar (D) word lists. Dashed lines: articulation during list presentation. Solid lines: no articulation (control).

articulatory suppression) and phonetic similarity of the list (similar vs. dissimilar) as within-subject factors. There was a large main effect of the articulation condition $[F(1,31)=115.62, p<.001, M S e=0.47]$ and of phonetic similarity $[F(1,31)=71.85, p<.001, M S \mathrm{e}=$ $0.46]$. There was also an interaction of these two variables $[F(1,31)=12.13, p<.002, M S e=0.28]$, suggesting that the suppression task interfered with performance more for the phonetically dissimilar lists, as expected. A rehearsal process that can be blocked by articulatory suppression appears to be more important for adults' recall of dissimilar than of similar lists.

The first column of Table 1 provides the means and two derived measures of performance that can be used to make further quantitative comparisons between the present results and the developmental findings of Hulme (1984). One measure is the difference in performance levels for phonetically dissimilar versus similar lists. Additionally, because it could be suggested that these difference scores are related to performance levels, with larger differences occurring at higher mean levels of performance (i.e., in the no-articulation control condition), a stricter, "decrement ratio" measure was obtained by dividing each difference score by the mean across both similar and dissimilar lists. For each measure in the table, an estimate of the age-equivalent of performance (the oldest age at which Hulme's subjects scored below the performance level shown) also is provided. All measures in Experiment 1 indicate that the age-equivalents of performance were reduced by the articulatory suppression task, which produced performance most like that of 5-year-olds.

This conclusion must be qualified by the observation that, in the control condition, adults still did not quite perform at a level equal to that of Hulme's oldest (10-yearold) subjects. The probable explanation is that there were order effects across the two articulation conditions for 
Table 1

Methods and Results of Four Experiments

\begin{tabular}{|c|c|c|c|c|c|}
\hline & Experiment 1 & Experiment 2 & Experiment 3 & \multicolumn{2}{|l|}{ Experiment 4} \\
\hline \multicolumn{6}{|c|}{ Method } \\
\hline$N$ & 32 & 32 & 32 & \multicolumn{2}{|c|}{36} \\
\hline Stimulus Delivery & Oral & Oral & Taped & \multicolumn{2}{|c|}{ Taped } \\
\hline Suppression Task & $\begin{array}{l}\text { Whisper } \\
\text { alphabet }\end{array}$ & $\begin{array}{l}\text { Whisper } \\
\text { list item }\end{array}$ & $\begin{array}{l}\text { Whisper } 1 \\
\text { letter after } \\
\text { each item }\end{array}$ & $\begin{array}{l}\text { (a) Whisper } \\
\text { I letter once }\end{array}$ & $\begin{array}{l}\text { (b) Whisper } \\
1 \text { letter } \\
\text { continuously }\end{array}$ \\
\hline Intended Effect & $\begin{array}{l}\text { Prevent } \\
\text { rehearsal }\end{array}$ & $\begin{array}{l}\text { Prevent } \\
\text { cumulative } \\
\text { rehearsal }\end{array}$ & $\begin{array}{l}\text { Replicate } \\
\text { Experiment } 1\end{array}$ & \multicolumn{2}{|c|}{ Reduce processing demands } \\
\hline \multicolumn{6}{|c|}{ Results } \\
\hline $\begin{array}{l}\text { Means-Control } \\
\text { Dissimilar } \\
\text { Similar } \\
\text { Difference* }\end{array}$ & $\begin{array}{l}5.59(9) \\
4.25(10) \\
1.34(8)\end{array}$ & $\begin{array}{l}5.75(10) \\
4.31(10) \\
1.44(8)\end{array}$ & $\begin{array}{l}5.72(10) \\
4.28(10) \\
1.44(8)\end{array}$ & $\begin{array}{l}5.8 \\
4.2 \\
1.5\end{array}$ & $\begin{array}{l}(10) \\
(10) \\
(10)\end{array}$ \\
\hline $\begin{array}{l}\text { Means-Suppression } \\
\text { Dissimilar } \\
\text { Similar } \\
\text { Difference* }\end{array}$ & $\begin{array}{l}3.97(4) \\
3.28(4) \\
0.69(5)\end{array}$ & $\begin{array}{l}4.69(5) \\
3.53(9) \\
1.16(8)\end{array}$ & $\begin{array}{l}4.00(4) \\
3.19(4) \\
0.81(5)\end{array}$ & $\begin{array}{l}4.81(7) \\
3.67(9) \\
1.14(8)\end{array}$ & $\begin{array}{l}4.86(8) \\
3.89(9) \\
0.97(5)\end{array}$ \\
\hline $\begin{array}{l}\text { Similarity Effect } F \\
d f\end{array}$ & $\begin{array}{c}71.85 \\
1,31\end{array}$ & $\begin{array}{c}101.551 \\
1,31\end{array}$ & $\begin{array}{c}62.78 \\
1,31\end{array}$ & $\begin{array}{c}102.36 \| \\
1,35\end{array}$ & $\begin{array}{c}92.58 \\
1,35\end{array}$ \\
\hline $\begin{array}{l}\text { Suppression Effect } F \\
\quad d f\end{array}$ & $\begin{array}{c}115.62 \| \\
1,31\end{array}$ & $\begin{array}{c}76.26 \| \\
1,31\end{array}$ & $\begin{array}{c}113.93 \| \\
1,31\end{array}$ & $\begin{array}{c}50.66 \| \\
1,35\end{array}$ & $\begin{array}{c}40.00 \\
1,35\end{array}$ \\
\hline $\begin{array}{l}\text { Interaction } F \\
\qquad d f\end{array}$ & $\begin{array}{c}12.13 \S \\
1,31\end{array}$ & $\begin{array}{c}1.18 \text { (n.s.) } \\
1,31\end{array}$ & $\begin{array}{c}5.58 \ddagger \\
1,31\end{array}$ & $\begin{array}{c}3.51 \text { (n.s.) } \\
1,35\end{array}$ & $\begin{array}{c}10.11 \S \\
1,35\end{array}$ \\
\hline \multicolumn{6}{|l|}{ Decrement Ratio† } \\
\hline Suppression & $0.19(5)$ & $0.28(8)$ & $0.23(5)$ & $0.27(8)$ & $0.22(5)$ \\
\hline
\end{tabular}

Note-Numbers in parentheses refer to the age-equivalent of performance-the oldest age at which Hulme's (1984) subjects scored no higher than the performance level shown. *Dissimilar span minus similar span. †Difference score (dissimilar - similar) divided by the mean of both conditions. $\quad \neq p<.05 . \quad \S p<.01$. $\| p<.001$.

adults in the present experiment, where Hulme's children were tested in one condition only. When the first condition in the present experiment is examined separately, the pattern of results is strengthened further. Specifically, when the control condition came first, the mean word spans for this condition were 5.81 for dissimilar lists and 4.13 for similar lists, causing all derived measures to surpass those for Hulme's oldest (10-year-old) subjects. In contrast, when the articulatory suppression condition came first, the means for that condition were $\mathbf{3 . 8 1}$ for dissimilar lists and 3.18 for similar lists, causing the derived measures to fall below those listed in Table 1. Inspection of each subject's first condition clearly reinforces the conclusion that articulatory suppression produces a much more childlike pattern of performance in adults. ${ }^{1}$

\section{EXPERIMENTS 2, 3, AND 4}

In the first experiment, both the phonetic similarity effect and performance levels were reduced by an articulatory suppression task that presumably blocked covert rehearsal and imposed an additional processing demand. The remaining experiments provided replications, and the results clarify the effects of articulatory suppression in at least two ways. First, they indicate that the complexity of the processing demands imposed by the suppression task helps to determine the extent of its effectiveness. Second, they suggest that the aspects of suppression that are detrimental to the phonetic similarity effect may not be the same aspects that decrease the overall level of performance.

In Experiment 2, subjects were to whisper each list item repeatedly as quickly as possible when it was presented, until the next list item was heard. Thus, although simple rehearsal was required, the cumulative, serial repetition of the list as a whole presumably was blocked. The methods and results of Experiment 2 (along with all of the others) are summarized in Table 1 and Figure 1. There was a moderate detrimental effect of articulation on the overall level of performance (not as large as the effect in Experiment 1), but the articulation condition $x$ phonetic similarity interaction that would indicate a suppression of the phonetic similarity effect was not significant. This suggests that there may be some flexibility in the types of covert speech behavior that will produce a phonetic similarity effect.

In Experiment 3, as in Experiment 2, subjects had to respond to each list item. In this experiment, however, the articulatory task was to repeatedly whisper " $a$ " after the first list item, " $b$ " after the second item, and so on, 
rather than whispering the list item. The effects of alphabetic recital observed in Experiment 1 were closely replicated (see Table 1 and Figure 1).

In order to rule out the possibility that the two-way interactions in Experiments 1 and 3 could have occurred because of a floor effect for performance in the articulatory suppression conditions, the data from the two experiments were combined and then split into halves according to each subject's mean performance across conditions. Confirming the original findings, the same patterns of effects were obtained for both the upper and lower split-halves. For the upper half, the means for the control condition were 6.34 for dissimilar lists and 4.63 for similar lists, whereas for the articulatory suppression condition the means were 4.56 for dissimilar lists and $\mathbf{3 . 6 3}$ for similar lists. A separate analysis of these scores, using the same factors as used in the main analyses, produced an effect of articulation condition $[F(1,31)=117.21, p<.001, M S e=$ $0.53]$ and of phonetic similarity $[F(1,31)=93.05$, $p<.001, M S \mathrm{e}=0.61]$, as well as an articulation condition $\times$ phonetic similarity interaction $[F(1,31)=9.85$, $p<.004, M S e=0.50$ ]. For the subjects with mean scores in the lower half of the sample, the pattern of results was similar (control: dissimilar lists, 4.97, similar lists, 3.91; articulatory suppression: dissimilar lists, 3.41, similar lists, 2.84). An analysis of these scores again yielded effects of articulation condition $[F(1,31)=111.15$, $p<.001, M S e=0.50]$ and phonetic similarity $[F(1,31)$ $=57.57, p<.001, M S \mathrm{e}=0.37]$, as well as an articulation condition $\times$ phonetic similarity interaction $[F(1,31)$ $=5.90, p<.02, M S e=0.34]$.

In all three of the experiments discussed so far, subjects had to continually change their articulations throughout each trial. In order to assess the impact of articulation with fewer additional speech planning and programming demands, we had the subjects in Experiment 4 repeat a single letter throughout the session. There were three groups of subjects, who were assigned the letters B, D, and F, respectively. (These are the first three letters of the alphabet that appear to be easy to pronounce repetitively.) In one suppression condition (a), the subjects were to whisper the letter only once following each list item; in another suppression condition (b), they were to whisper the letter continuously throughout the list presentation. Each subject received these two conditions and a control condition in a counterbalanced order. The results are shown in the last panel of Figure 1 and the last two columns of Table 1. The overall level of performance was reduced moderately by both suppression conditions, relative to the control condition, but the phonetic similarity effect differed in these two conditions. The phonetic similarity $\times$ articulation condition interaction within an overall ANOVA was significant, so separate analyses with each suppression condition and the control (shown in Table 1) were carried out to determine the locus of the interaction. The interaction was significant only when the suppression task was continuous. This conclusion is supported by the derived measures in Table 1, which indicate that there was a sub- stantial reduction in the age-equivalent of the phonetic similarity effect for suppression task b only.

\section{GENERAL DISCUSSION}

The main outcome of this set of experiments was that articulatory suppression in adults decreased both the level of performance and the relative advantage for phonetically dissimilar items, to such an extent that performance in some conditions was comparable to what has been obtained in 5-year-old children (Hulme, 1984) without articulatory suppression. These results provide convergent support for the conclusion of Hayes and Rosner (1975) that covert rehearsal is largely responsible for the developmental change in this type of memory task. This hypothesis also is strengthened by considerable evidence that rehearsal processes develop during childhood (Flavell, Beach, \& Chinsky, 1966), and that rehearsal training can result in improved performance in young children (Bebko, 1979; Keeney, Cannizzo, \& Flavell, 1967; Kingsley \& Hagen, 1969).

In the present experiments we also investigated which aspects of articulatory suppression alter adults' memory performance. All types of suppression substantially and significantly reduced the overall level of recall. The largest decrements in both the performance level and the magnitude of the phonetic similarity effect occurred when the subjects had to whisper an alphabetic sequence (Experiments 1 and 3). Nevertheless, merely articulating one letter over and over again (Experiment 4, suppression Task b) significantly reduced the magnitude of the phonetic similarity effect. This was not the case, however, with a single repetition of one letter following each list item (Experiment 4, suppression Task a) or when the subjects carried out noncumulative rehearsal (in Experiment 2$)^{2}$

Despite these findings, the exact aspects of covert speech necessary to obtain a phonetic similarity effect in recall are uncertain; the results rule out some hypothetical possibilities but do not specify a unique mechanism. If one considers only the results of Experiment 1, there are at least four general ways in which the articulatory suppression task could interfere with performance: by preventing covert recitation of the list items; by preventing the premotor planning of speech responses; by preventing any aspect of speech coding, other than rehearsal, that requires attention and effort (possibly including premotor planning); or by introducing extraneous, alphabetic information.

The results of Experiments 2-4, however, impose additional constraints. First, a phonetic similarity effect seems to have emerged in much the same way no matter whether the suppression task included a single speechmotor routine or multiple routines. Specifically, only one routine would be needed to repeat a single letter in Experiment 4 , it is not clear whether one or many routines would be needed to recite the alphabet in Experiment 1, and multiple routines probably were needed to utter the 
next letter of the alphabet following each list item in Experiment 3. Yet the amount of suppression of the phonetic similarity effect was comparable in each of these three articulatory conditions (see the decrement ratio measure in Table 1). Also, the reduction in processing demands in Experiment 4 did not alter the suppression effect, provided that articulation was continuous. However, the simple interruption of prespeech planning and the insertion of alphabetic material in suppression Task a was not sufficient to suppress the similarity effect.

Taken together, the results suggest that articulatory suppression reduces the magnitude of the phonetic similarity effect by blocking a covert, speech-related process that requires at most a limited amount of effort in adults and can be successfully carried out in intermittent intervals between list-item presentations. Covert, cumulative rehearsal may be only one of several candidate processes fitting this description; however, it is a candidate that is made more appealing by the successful induction, using rehearsal training, of the similarity effect in young children (Hayes \& Rosner, 1975).

On the other hand, in the present study the phonetic similarity effect was never suppressed to a point equivalent to the effect in 4-year-old children. It is possible that the suppression task was not totally effective in eliminating covert rehearsal. However, another explanation of this residual effect of phonetic similarity is that there is a second source of developmental change that contributes to the effect. For example, children's representation of phonetic sequences is enriched as the children develop (Cowan \& Kielbasa, 1986), and in particular, as they learn to read (Morais et al., 1979; Read, 1978; Treiman, 1985). This enhanced representation could lead to an increase in confusions in memory stemming from phoneme identities among words (cf. Treiman \& Breaux, 1982).

There are several theoretical benefits to be derived from the present study. First, it may assist in an understanding of the role of covert speech processes in the normal development of memory. Specifically, it provides convergent evidence (along with rehearsal-training experiments with children) that developing covert speech processes contribute to performance primarily for lists of phonetically dissimilar items. Second, it may lead to a better understanding of developmental abnormality, inasmuch as the phonetic similarity effect has been found to be reduced in children with poor reading ability (Mann, 1984; Mann, Liberman, \& Shankweiler, 1980; Shankweiler, Liberman, Mark, Fowler, \& Fischer, 1979) and other learning disabilities (Siegel \& Linder, 1984). Third, this study may aid in an understanding of performance in a limited number of real-world tasks that in some ways resemble the current task. For example, orally spelled words can include letters with names that are phonetically similar (e.g., $d-e-b-t)$, dissimilar (e.g., $b-o-w-l)$, or in between (e.g., $b-e-a-k$ ), and this could be one factor in children's memorization or transcription of the spelling of various words. A final, more speculative suggestion is that, in certain circumstances, adults receiving articulatory suppression tasks might be used as "model children" in order to predict how actual children will respond to various stimulus conditions in speech memory tasks. This approach, in which an individual's performance is analyzed into the effects of multiple mnemonic mechanisms acting together, may become more feasible as our understanding of particular recall mechanisms advances.

\section{REFERENCES}

Alegria, J., \& Pignot, E. (1979). Genetic aspects of verbal mediation in memory. Child Development, 50, 235-238.

BeBko, J. M. (1979). Can recall differences among children be attributed to rehearsal effects? Canadian Journal of Psychology, 33, 96-105.

ConRad, R. (1971). The chronology of the development of covert speech in children. Developmental Psychology, 5, 398-405.

Cowan, N., Braine, M. D. S., \& LeavitT, L. A. (1985). The phonological and metaphonological representation of speech: Evidence from fluent backward talkers. Joumal of Memory \& Language, 24, 679-698.

Cowan, N., \& Kielbasa, L. (1986). Temporal properties of memory for speech in preschool children. Memory \& Cognition, 14, 382-390.

Cowan, N., Suomi, K., \& Morse, P. A. (1982). Echoic storage in infant perception. Child Development, 53, 984-990.

Flavell, J. M., Beach, D. R., \& Chinsky, J. M. (1966). Spontaneous verbal rehearsal in a memory task as a function of age. Child Development, 37, 283-299.

HAYES, D. S., \& RoSNER, S. R. (1975). The phonetic effect in preschool children: The influence of overt rehearsal and verbal instructions. Journal of Experimental Child Psychology, 20, 391-399.

Hulme, C. (1984). Developmental differences in the effects of acoustic similarity on memory span. Developmental Psychology, 20, 650-652

Irwin, R. J., Ball, A. K. R., Kay, N., \& Stillman, J. A. (1985). The development of auditory temporal acuity in children. Child Development, 56, 614-620.

Keeney, T. J., Cannizzo, S. R., \& Flavell, J. H. (1967). Spontaneous and induced verbal rehearsal in a recall task. Child Development, 38, 953-966.

KingsLey, P. R., \& Hagen, J. W. (1969). Induced versus spontaneous rehearsal in short-term memory in nursery school children. Developmental Psychology, 1, 40-46.

LEVY, B. A. (1971). Role of articulation in auditory and visual shortterm memory. Journal of Verbal Learning \& Verbal Behavior, 10, 123-132.

ManN, V. A. (1984). Reading skill and language skill. Developmental Review, 4, 1-15.

Mann, V. A., Liberman, I. Y., \& Shankweiler, D. (1980). Children's memory for sentences and word strings in relation to reading ability. Memory \& Cognition, 8, 329-335.

Morais, J., Carey, L., Alegria, J., \& Bertelson, P. (1979). Does awareness of speech as a sequence of phones arise spontaneously? Cognition, 7, 323-331.

MURRAY, D. J. (1967). The role of speech responses in short-term memory. Canadian Journal of Psychology, 21, 263-276.

MuRRAY, D. J. (1968). Articulation and acoustic confusability in shortterm memory. Journal of Experimental Psychology, 78, 679-684.

Peterson, L. R., \& Johnson, S. T. (1971). Some effects of minimizing articulation on short-term retention. Journal of Verbal Learning \& Verbal Behavior, 10, 346-354.

READ, C. (1978). Children's awareness of language, with emphasis on sound systems. In A. Sinclair, R. J. Jarvella, \& W. J. M. Levelt (Eds.), The child's conception of language. Berlin: Springer-Verlag. 
Shankweiler, D., Liberman, I., Mark, L., Fowler, C., \& FISCHER, F. (1979). The speech code and learning to read. Journal of Experimental Psychology: Human Learning \& Memory, 5, 531-545.

SIEGEL, L., \& LiNDER, B. (1984). Short-term memory processes in children with reading and arithmetic learning disabilities. Developmental Psychology, 20, 200-207.

SIPE, S., \& ENGLE, R. W. (1986). Echoic memory processes in good and poor readers. Journal of Experimental Psychology: Learning, Memory, \& Cognition, 12, 402-412.

Treiman, R. (1985). Phonemic awareness and spelling: Children's judgments do not always agree with adults'. Joumal of Experimental Child Psychology, 39, 182-201.

Treiman, R., \& Breaux, A. M. (1982). Common phoneme and overall similarity relations among spoken syllables: Their use by children and adults. Journal of Psycholinguistic Research, 11, 569-598.

\section{NOTES}

1. As in the developmental experiments after which the present work was modeled, the repeated sampling of list stimuli from eight-item sets could lead to interference between lists, and this interference could be greater for similar than for dissimilar lists. This interlist confusion potentially could occur more readily for adults than for children, which would detract from the comparability of the adult and developmental work. However, for two reasons, interlist confusions do not interfere with the present results: (1) Interlist confusions could increase the magnitude of the phonetic similarity effect, but there is no obvious way in which they could alter the effects of articulatory suppression, which is the main focus of the present study. Any component of the similarity effect based on interlist confusion should be present even in the suppression conditions; the reduction of the similarity effect in the suppression conditions to a level similar to that of 5-year-olds may have occurred in spite of interlist confusions, but not because of them. (2) The data for the control condition of the present experiment closely matched the data for Hulme's (1984) oldest group of children. If interlist confusions for similar lists were a greater factor for adults, performance on these lists would have been lower. It is true that each of the present experiments included two or three conditions per subject rather than just one, but similar results were obtained when the first condition from every subject was examined alone (see text). The similarity of the present data to those obtained for Hulme's oldest group of children is a strength of the present work.

2. An interesting aspect of these results is that it is possible to dissociate the effects of articulation on the overall level of performance from effects on the magnitude of the phonetic similarity effect. In the developmental data (Conrad, 1971; Hulme, 1984), the two effects are closely related; a lower level of performance in younger children is accompanied by a smaller phonetic similarity effect. One might suppose that the similarity effect is somehow a consequence of the level of recall, but the decrement ratio measure presented in Table 1 argues against this supposition. For example, the ratios were nearly identical for the suppression condition of Experiment 3 (in which the mean performance level across lists was 3.60) and the single-letter, continuous-repeat condition of Experiment 4 (in which the mean performance level was 4.38). Also compare the two conditions of Experiment 2.

(Manuscript received February 9, 1987; revision accepted for publication May 18, 1987.) 